\title{
LA BÚSQUEDA DE LA VERDAD EN LA TRADICIÓN PRAGMATISTA*
}

\author{
Jaime Nubiola \\ Universidad de Navarra, España
}

"Si uno dice la verdad, tarde o temprano será descubierto."

Oscar Wilde ${ }^{1}$

\section{Introducción}

El tema de la verdad es una cuestión enrevesada, en la que se entrecruzan buena parte de los puzzles o de los debates que atraviesan la filosofía, la ciencia y la cultura de nuestro tiempo. Nos encontramos en una sociedad que vive en una amalgama imposible de un escepticismo generalizado acerca de los valores y un supuesto fundamentalismo cientista acerca de los hechos. Se trata de una mezcolanza de una ingenua confianza en la Ciencia con mayúscula y de aquel relativismo perspectivista que expresó el poeta Campoamor con su "nada hay verdad ni mentira; todo es según el color del cristal con que se mira"2. Para ilustrar esto basta con hojear cualquier periódico o comprobar cómo sigue repitiéndose a los alumnos de periodismo que han de distinguir entre información y opinión, entre hechos y valoraciones, o escuchar a los políticos (al menos en mi país) que aseguran que las opiniones son libres o que todas merecen igual respeto. Aunque sea cómodo para quien detenta el poder esa separación entre ciencia y valores, mantener un desgarro así entre lo fáctico y lo normativo resulta a la postre insoportable. Los seres humanos anhelamos una razonable integración de las diversas facetas de las cosas; la contradicción flagrante desquicia nuestra razón, hace saltar las bisagras de nuestros razonamientos y bloquea el diálogo y la comunicación.

\footnotetext{
* El origen de estas páginas se encuentra en la invitación a impartir un curso de postgrado en la Universidad Católica de Santa $\mathrm{Fe}$ en septiembre de 1998. En el marco de ese curso impartí esta conferencia que se publica ahora por primera vez.

${ }^{1}$ Wilde, O., Frases y citas filosóficas para el uso de los jóvenes, en El arte del ingenio, Valdemar, Madrid, 1995, p. 177.

${ }^{2}$ Campoamor, R., Obras poéticas completas, Aguilar, Madrid, 1972, p. 148.
} 


\section{La verdad buscada}

He elegido la búsqueda de la verdad como foco de estas páginas porque la verdad es primordialmente aquello que los hombres (y mujeres) de ciencia anhelamos y buscamos, y porque es - tal como veo yo las cosas - la manera más adecuada para comprender la tradición pragmatista. Me gusta destacar que la verdad buscada es la verdad objetiva; es la verdad objeto de los afanes compartidos en el espacio y en el tiempo de cuantos han dedicado sus vidas a saber y a generar nuevos conocimientos. Quienes empeñamos nuestras vidas en saber no lo hacemos por afán de poder ni mucho menos por obtener unas patentes o escribir unos libros que nos hagan millonarios, sino que lo que nos mueve realmente es el saber mismo: nuestras vidas están animadas por el deseo de averiguar la verdad, por el "impulso - escribió Peirce ${ }^{3}$ — de penetrar en la razón de las cosas". Como ha escrito Polo, es la verdad la que encarga la tarea al pensar. La inteligencia se pone en marcha para ver si puede articular un discurso que esté de acuerdo con la verdad ${ }^{4}$.

Frente al diagnóstico postmoderno de Rorty que aboga por la disolución de la filosofía en la literatura, en la conversación general de la humanidad (y que así se manifiesta en amplias áreas de la filosofía académica tanto europea como americana) y frente al fundacionalismo cientista de los herederos del Círculo de Viena (todavía dominante en la tradición analítica), deseo exponer un camino intermedio, con pretensiones quizá más modestas, pero que por estar anclado en la experiencia personal de cada uno y en la experiencia colectiva de la especie humana, afronta mejor el reto de dar razón del progreso histórico de la verdad, el reto de comprender el sentido del crecimiento de los saberes y el proceso de generación de nuevos conocimientos. Adoptar esta perspectiva significa destacar que la búsqueda de la verdad no es un problema "teórico", sino genuinamente práctico, pragmático. Quizá sea conveniente advertir que "Pragmático" es una palabra peligrosa, pues es usada con frecuencia como insulto entre políticos y periodistas, pero en estos finales del siglo XX que ha visto tantas maneras diversas -y a menudo opuestas- de tematizar filosóficamente la verdad, el enfoque pragmatista, que se atiene preferentemente a la experiencia efectiva de la práctica científica y vital humanas, ocupa -me parece- un lugar primordial. Quizá por esa razón en la reciente compilación Teorías de la verdad en el siglo $X X$ en el que se reúnen veintisiete de los más importantes ensayos de nuestro siglo sobre la ver-

3 Peirce, C.S., Collected Papers of Charles Sanders Peirce. editado por C. Hartshorne, P. Weiss, y A. Burks, Harvard University Press, Cambridge. MA, 1936-58, 1.44, c.1896 (En adelante, CP seguido de número de volumen y parágrafo y año).

${ }^{4}$ Polo, L., Introducción a la filosofia, Eunsa, Pamplona,1995, p. 21. 
dad, sus editores dedican la primera sección a las teorías pragmatistas ${ }^{5}$ y destacan entre ellas el enfoque de Susan Haack en cuya dirección puede situarse este trabajo ${ }^{6}$.

Como ha escrito Alejandro Llano ${ }^{7}$, "la filosofía no siempre había concedido a la verdad práctica la atención que merece. Pero sólo es viable rehabilitarla cuando no se extrapola. Porque cuando el valor de la praxis humana se absolutiza el valor de la verdad se disuelve". Absolutizar el valor de la praxis sería afirmar con el pragmatismo vulgar que la verdad es meramente algo fabricado por los seres humanos, y en ese sentido algo arbitrario, relativo y por tanto a fin de cuentas, de escaso o ningún valor. Lo que quiero afirmar más bien es que las verdades se descubren y se forjan en el seno de nuestras prácticas comunicativas; que la verdad -como dejó escrito Platón ${ }^{8}$ - se busca en comunidad; "que como me decía un día Guy Debrock- no hay verdad fuera de la búsqueda, aunque no es la búsqueda la que causa la verdad".

En estos últimos años asistimos a un ataque frontal contra la semántica lógica, esto es, contra la semántica que aspira a construir modelos teóricos con la pretensión de explicar cómo funciona el lenguaje. La acusación no consiste sólo en decir que una aproximación lógica al lenguaje es demasiado abstracta, sino también en que el propio formalismo teórico resulta inapropiado para modelar el mundo real ${ }^{9}$. El ataque principal contra la semántica de modelos estriba en que las nociones de verdad y referencia que emplea son "objetivistas", no tienen en cuenta el modo en que la mente humana contribuye a conformar el mundo tal como lo vemos. Frente a las versiones objetivistas de la verdad que nacen del antipsicologismo fregeano y del empirismo del Círculo de Viena, en los últimos años ha irrumpido con fuerza -es decir, con capacidad explicativa- en el seno de la filosofía analítica una concepción de la verdad en la que ésta no trasciende el lenguaje, sino que se halla inserta en nuestro conocimiento y en nuestros medios de reconocimiento del ajuste entre lă expresiones lingüísticas y el mundo. Afirmar que la verdad está maclada con nuestro lenguaje no es degradarla o rebajarla, sino que es destacar su humanidad, su efectivo enraizamiento en nuestras prácticas comunicativas y nuestros objetivos vitales. La verdad, toda la verdad y

\footnotetext{
${ }^{5}$ Nicolás, J.A. y Frápolli, M. J. (eds.), Teorías de la verdad en el siglo XX, Tecnos, Madrid, 1997.

${ }^{6}$ Haack, S, "El interés por la verdad: qué significa, por qué importa", en Teorias de la verdad en el siglo XX, Tecnos, Madrid, 1997, pp. 53-62; "La ética del intelecto: Un acercamiento peirceano", Anuario Filosófico 29 (1996), pp. 1413-1433; y especialmente Evidencia e investigación, Tecnos, Madrid, 1997.

${ }^{7}$ Llano, A., Gnoseología, Eunsa, Pamplona 1983, 32.

${ }^{8}$ Fedón, 99d.

${ }^{9}$ Lakoff, G., Women, Fire, and Dangerous Things, University of Chicago Press, Chicago, 1987.
} 
nada más que la verdad es un camello lógico, que no puede pasar siquiera por el ojo de un gramático ${ }^{10}$.

Con Hilary Putnam -y con una gran tradición de pensadores antes que él- me gusta distinguir entre la Verdad con mayúscula y las verdades que los hombres forjamos. Estas últimas, las verdades que los seres humanos han conquistado laboriosamente mediante su pensar son resultado de la historia: Veritas filia temporis, repetían los escolásticos citando al historiador romano Aulo Gelio $(125-175)^{11}$. Que la verdad sea hija del tiempo significa también que la verdad futura depende de nuestra libre actividad, de lo que cada uno contribuyamos personalmente al crecimiento de la humanidad, al desarrollo y expansión de la verdad $^{12}$. La verdad con minúscula no ha sido descubierta de una vez por todas, sino que es un cuerpo vivo que crece y que está abierto a la contribución de todos. Con el dicho medieval, somos enanos a hombros de gigantes ${ }^{13}$, pero también -como decía con fuerza el humanista Juan Luis Vives rectificando a Juan de Salisbury- "ni somos enanos, ni fueron ellos gigantes, sino que todos tenemos la misma estatura"14. En esta expresión del Renacimiento humanista se refleja bien el estilo democrático, pluralista, que se encuentra también en el centro de la aproximación pragmatista más reciente, anclada en la convicción de que en cada genuino esfuerzo intelectual hay algún aspecto luminoso del que podemos aprender, de que la verdad humana es el saber acumulativo construido entre todos mediante una historia multisecular de intentos, errores, rectificaciones y aciertos.

\section{La noción de verdad}

El núcleo "intuitivo" de la noción de verdad está conformado por tres elementos que interactúan entre si $^{15}$. Llamamos verdadero en primer lugar a lo que está realmente presente contraponiéndolo a lo imaginario, a lo irreal: se trata de la dimensión que conecta lo verdadero con lo que es, con lo real, destacada por la raíz griega de la verdad (aletheia) como lo patente. En segundo lugar, consideramos verdadero a lo fiable y falso a aquello de lo que no podemos fiarnos: enlaza con la noción de autenticidad y con la raíz latina veritas y se traduce

\footnotetext{
${ }^{10}$ Austin, J. L., Philosophical Papers, Oxford University Press, Oxford, $2^{\mathrm{a}}$ ed., 1970, 117.

${ }^{11}$ Gelio, A., Noctium Atticarum, XII, 11, p. 2.

12 Mondolfo, R., "Veritas Filia Temporis in Tommasso d'Aquino", Momenti del Pensiero Greco e Cristiano, Morano, Nápoles, 1964, pp. 21-36.

13 Jeaunneau, E., Nani sulle Spalle di Giganti, Guida, Nápoles, 1969.

${ }^{14}$ Vives. J.L., Opera Omnia, edición de G. Mayans. Benedicto Monfort, Valencia, pp, 1782-1790, VI, 39.

${ }^{15}$ Cfr. J. A. Nicolás y M. J. Frápolli: "Presentación", en Teorias de la verdad en el siglo XX, 11.
} 
en confianza (fides) con las personas o con las cosas. Esta es la dimensión de la verdad que privilegia la tradición hebrea al destacar el valor del testimonio y su autoridad como fuente del conocimiento ${ }^{16}$. El tercer elemento es la idea de adecuación, de ajuste, entre lo que se dice o piensa y lo que acontece o se hace. $\mathrm{Su}$ ámbito natural es el lenguaje, en particular sus recursos para evaluar o medir la capacidad de las palabras para expresar con claridad el pensamiento y para reflejar con precisión las cosas.

En cierta manera, acentuar la dimensión pragmática significa focalizar la reflexión más en torno al par verdad/mentira que al par verdad/falsedad que ha estado más en el centro de la aproximación cientista dominante en la cultura contemporánea. La mentira nos resulta, por así decir, mucho más humana que la falsedad porque apunta más directamente al ámbito comunicativo de la verdad. San Agustín cuenta en Las confesiones que, aunque en su vida había tratado a muchos mentirosos, nunca había conocido a nadie que deseara ser engañado. En cierto sentido, quienes no desean ser engañados aman ya la verdad y tienen por tanto alguna noticia de ella ${ }^{17}$. Con ello estoy afirmando de nuevo la tesis de que el mejor marco para adentrarse en el análisis de la verdad es identificando la verdad, no como el resultado sofisticado de una teoría lógica, sino como aquello que siempre buscamos. Me parece que esta perspectiva se encuentra en sintonía con la experiencia práctica de cualquiera que se dedique a la docencia. El factor decisivo del crecimiento de los estudiantes es siempre su afán de verdad, la voluntad de saber, que convierte ese anhelo natural en la aventura vitalmente más placentera: "el género humano vive de arte y de razones", recuerda Tomas de Aquino $^{18}$ al comentar aquel pasaje de Aristóteles en el arranque de la Metafisica.

De acuerdo con todo este marco, querría ahora centrar la atención en tres autores de singular relieve en la filosofía de nuestro siglo. Comienzo por Wittgenstein, pues muy probablemente es el más conocido, abordo luego el pensamiento de Peirce sobre esta cuestión y termino finalmente con Hilary Putnam.

\section{Ludwig Wittgenstein (1889-1951)}

Como decía Wittgenstein a la Prof. Anscombe casi a modo de despedida a las puertas de la muerte en la primavera de 1951: "Elisa, yo siempre he

\footnotetext{
${ }^{16}$ Cfr. Artola, A.M., "La verdad en la Biblia", en A. M. Artola y J. M. Sánchez Caro, Biblia y palabra de Dios, Verbo Divino, Estella, 1990, 216-217.

${ }^{17}$ San Agustín, Confesiones, X, 23, 33. Cfr. C. Cardona: "Querer la verdad", Escritos Arvo, n 128, XII (1992); Juan Pablo II: Fides et ratio, 1998, n. 25.

${ }^{18}$ Aristóteles, Metafísica I, 1; Tomás de Aquino, Comentario a los Segundos Analíticos de Aristóteles, Proemio, 1.
} 
amado la verdad". Uno de los atractivos de Ludwig Wittgenstein radica en que su reflexión es el punto de partida o la fuente de inspiración de dos de las tradiciones dominantes en la filosofía de nuestro siglo. Así como el Tractatus LogicoPhilosophicus de 1921 fue considerado por Schlick el punto de viraje decisivo de la filosofía contemporánea y se convirtió -a pesar de Wittgenstein- en el libro de cabecera del positivismo lógico, sus Investigaciones Filosóficas, publicadas póstumamente en 1953 como compilación de buena parte de las reflexiones que le ocuparon en las dos últimas décadas de su vida, significaron la recuperación en el ámbito filosófico angloamericano de muchos de los temas y problemas del pragmatismo americano de finales del siglo XIX y las primeras décadas del XX.

Como ha señalado Donald Davidson ${ }^{19}$, -ésta es una de las tesis centrales de mi exposición- Wittgenstein nos puso en el camino real para encontrar en la comunicación interpersonal el origen de la noción de verdad objetiva. Si el lenguaje es esencial al pensamiento y si estamos de acuerdo con Wittgenstein en que no puede haber lenguaje privado, y en que sólo la comunicación con los demás nos proporciona el uso correcto de las palabras, entonces, de la misma manera y con la misma rotundidad, ha de afirmarse, por una parte, que no puede haber pensamiento privado -un pensamiento con un contenido proposicional que sólo yo pueda entender- $y$, por otra, que la comunicación interpersonal es la que proporciona la pauta de objetividad en el ámbito cognoscitivo.

En este sentido, estoy afirmando que la objetividad de la verdad está marcada con el carácter público del pensamiento, con el carácter solidario, social, del lenguaje y con el carácter racional de la realidad. Los tres elementos pensamiento, lenguaje y realidad- que intervienen en la discusión acerca de la verdad se confieren sentido respectivamente en su interrelación, y esa constelación de sentido se establece mediante la comunicación interpersonal. No hay pensamiento privado, no hay lenguaje privado, no hay realidades privadas, no hay racionalidad privada: no hay privacidad. O mejor dicho, la concepción individualista de los seres humanos como agentes privados, puesta en boga por Descartes y el racionalismo moderno, distorsiona tanto lo que somos los seres humanos singulares que torna imposible la efectiva comprensión de nuestras relaciones comunicativas. El modelo racionalista nos convierte en fantasmas en la máquina incapaces de dar razón acerca de cómo acaecen los procesos de comunicación entre nosotros, entre los que se inscribe el descubrimiento de la verdad, y nuestras reflexiones sobre ella.

\footnotetext{
${ }^{19}$ Davidson, D., "Three Varieties of Knowledge", en A. Phillips Griffiths (ed.): A. J. Ayer Memorial Essays, Cambridge University Press, Cambridge, 1991, 159-60.
} 
Ante la pregunta reiterada acerca del porqué, como a veces suelen hacer los niños (o hacemos los filósofos, pues la filosofía -gusta decir Putnam- es educación para adultos), llega un momento en que la única respuesta que nos queda es el "yo hago así", "esto es lo que hago", o incluso con más propiedad "nosotros hacemos así". Nuestra forma de vida es la razón última que asiste a nuestra conducta. No se trata de buscar una explicación racional más profunda -que siempre puede ser de nuevo cuestionada- sino que nuestra racionalidad se apoya en nuestra vida. En este sentido la verdad o falsedad de un enunciado está enraizada ( grounded) en una forma de vida. El lecho rocoso de la verdad (bedrock of truth) de nuestras palabras es nuestra forma de vida. La verdad -in-cluidas las mediciones, la evaluación cuantitativa- es relativa, pero no relativa respecto de la voluntad o el capricho de quien habla o escribe, sino más bien respecto del juego lingüístico, de las prácticas y propósitos en los que dicha expresión está inserta y en los que cobra sentido ${ }^{20}$. Nuestras palabras no están asociadas mágicamente con las cosas sin nuestra intervención ${ }^{21}$, sino que en nuestras palabras se articulan nuestra personal biografía, la experiencia vital de cada uno, con el saber histórico de la humanidad. La verdad no es un problema teórico: los hechos y los valores, las teorías y las interpretaciones están interpenetrados con nuestras prácticas y objetivos vitales.

\section{Charles S. Peirce (1839-1914)}

La perspectiva que acabo de describir muy sumariamente tiene su origen principal en Charles S. Peirce. "La mayoría de la gente jamás ha oído hablar de él, pero lo oirá", dejó escrito el novelista norteamericano Walker Percy y parece que aquella profecía está comenzando a cumplirse. Durante décadas la figura y el pensamiento de Charles S. Peirce (1839-1914) han estado prácticamente relegados al olvido, pero desde finales de los setenta hay un estallido de interés y se ha puesto en marcha toda una industria editorial a su alrededor, promovida en especial por pensadores europeos como Karl-Otto Apel, Umberto Eco y Jürgen Habermas; y en el ámbito americano por Richard Bernstein, Hilary Putnam y una amplia comunidad de Peirce's scholars, dispersos por los Estados Unidos y el resto del mundo, pero conectados por correo electrónico.

En estos últimos años la figura de Charles S. Peirce está adquiriendo una relevancia creciente en muy distintas áreas del $\operatorname{saber}^{22}$, y su influencia sigue

\footnotetext{
${ }^{20}$ Boghossian, P.G. y Drewniak, E., "Wittgenstein and Peirce on Meaning", Diálogos 65 (1995), p. 181.

${ }^{21}$ Santambrogio, M. y Violi, P., "Introduction", en U. Eco, U.; Santambrogio, M. y Violi, P., Meaning and Mental Representations, Indiana University Press, Bloomington, IN, 1988, 18.

${ }^{22}$ Fisch, M., "The Range of Peirce's Relevance", The Monist 63 (1980), pp. 269-76; 64 (1981), pp.
} 
todavía creciendo ${ }^{23}$ : en astronomía, metrología, geodesia, matemáticas, lógica, filosofía, teoría e historia de la ciencia, semiótica, lingüística, econometría y psicología. En todos estos campos Peirce es considerado un pionero, un precursor o incluso como un "padre" o "fundador" (de la semiótica, del pragmatismo). Es muy común encontrar evaluaciones generales como la de Russell: "sin duda alguna (...) fue una de las mentes más originales de fines del siglo XIX y ciertamente el mayor pensador norteamericano de todos los tiempos" ${ }^{24}$ o la de Popper que lo describió como "uno de los más grandes filósofos de todos los tiempos"

Uno de los factores que aumentan el interés por el pensamiento de Peirce (y su competencia para el tema que me ocupa) es su personal participación en la comunidad científica de su tiempo, aunada a un sólido conocimiento de la filosofía de Kant y de la tradición escolástica, en particular de Duns Escoto, cualidades éstas no fáciles de encontrar entre científicos de primera línea. Me parece acertado caracterizar a Peirce -siguiendo en parte a Hookway ${ }^{26}$ - como un filósofo tradicional y sistemático, pero que aborda los problemas modernos de la ciencia, la verdad y el conocimiento desde una valiosa experiencia personal como lógico e investigador experimental en el seno de la comunidad científica internacional. Su buen conocimiento de la historia de la ciencia y de la historia de la filosofía otorgan un inusitado interés a sus esfuerzos por proporcionar una cartografía general de la metodología científica que resulta del todo relevante para la cuestión de la verdad.

Peirce consideró la búsqueda de la verdad como una tarea colectiva, y no como una búsqueda individual de fundamentos, según la imagen tradicional del filósofo solitario. Frente al fundamentalismo individualista, Peirce (y con él toda la tradición pragmatista) identificó la comunidad de los investigadores como el medio vital para el descubrimiento de la verdad y el crecimiento de la racionalidad científica $(C P 5.311,1868)$. Para Peirce, el florecimiento de la razón científica sólo acontece en las comunidades de investigación, en aquellos espacios en que la búsqueda de la verdad es una tarea cooperativa y corporativa. La introducción de nuevas ideas y su comprobación es una tarea que ha de desarrollarse cooperativamente, sometiendo al libre escrutinio de los demás las ideas propias y las evidencias en que se basa nuestra aceptación. Un marco de referencia como el que acabo de describir "no sólo desafía la apelación típicamente

123-41.

${ }^{23}$ Von Wright, G., The Tree of Knowledge and Other Essays, Brill, Leiden, 1993, 41.

${ }^{24}$ Russell, B., Wisdom of the West, Doubleday, Garden City, NY, 1959, 276.

${ }^{25}$ Popper, K., Objective Knowledge: An Evolutionary Approach, Clarendon Press, Oxford, 1972, p. 212.

${ }^{26}$ Hookway, C., Peirce, Routledge \& Kegan Paul. Londres, 1985, 1-3, 120. 
cartesiana a los fundamentos, sino que además esboza -ha escrito Bernstein ${ }^{27}$ una comprensión alternativa acerca del saber científico sin tales fundamentos".

A mí me gusta pensar que la metáfora del Árbol de la Ciencia -que campea en el escudo de muchas universidades y centros de investigación- proporciona una pista para recuperar la unidad vital del saber, porque expresa de modo gráfico que la ciencia es -en palabras de Peirce- "una entidad histórica viva" ( $C P$ 1.44, c.1896), "un cuerpo de verdad creciente y vivo" ( $C P$ 6.428, 1893). "Es totalmente verdad -escribe Peirce a Lady Welby- que nunca podemos alcanzar un conocimiento de las cosas tal como son. Podemos conocer sólo su aspecto humano. Pero ése es todo el universo que existe para nosotros" ${ }^{28}$. La ciencia se construye a lo largo del tiempo entre las opiniones mías y las de la comunidad en la que mi lenguaje, cultura y pensamiento se integran. En ese proceso avanzamos hacia la verdad mediante el diálogo con los demás, pues la comunidad es esencialmente una comunidad de diálogo y conversación.

En esta perspectiva, Peirce puede ser entendido como un continuador de la consideración trascendental de la objetividad en sentido kantiano, pero no tanto por las reglas que rigen el proceso de investigación, como por el contexto comunitario en el que los nuevos conocimientos se generan. Para el científico Charles S. Peirce la comunidad por excelencia es la de los investigadores, a la que el científico individual subordina su trabajo e interés particulares. La verdad está al final de un prolongado proceso de búsqueda esforzada con tal de que la investigación colectiva se prosiga lo suficiente: la verdad sería la "opinión final" de la comunidad científica, hacia la que necesariamente convergen los pareceres diversos. Esta verdad no es un ideal regulador inalcanzable sino que es a lo que necesariamente llegaría la comunidad investigadora si prosiguiera de modo sistemático su trabajo riguroso y creativo.

Cualquier presentación sumaria de Peirce no puede hacer justicia a la riqueza, complejidad y equilibrio de su posición. Como ha señalado Misak, Peirce logra establecer una posición que evita la consideración de la verdad como algo que trasciende todas las perspectivas y al mismo tiempo evita convertir la verdad en algo relativo a las diferentes perspectivas particulares. La clave se encuentra en que no desvincula la verdad de la búsqueda, ni vincula la verdad al resultado de algunas investigaciones determinadas ${ }^{29}$. Frente a la tradición carte-

\footnotetext{
${ }^{27}$ Bernstein, R., Beyond Objectivism and Relativism: Science, Hermeneutics, and Praxis, Blackwell, Oxford, 1983, pp. 71-72.

${ }^{28}$ Carta de 20 mayo 1911, en Hardwick, C., Semiotic and Significs: The Correspondence Between Charles S. Peirce and Victoria Lady Welby, Indiana University Press, Bloomington, IN, 1977, p. 141.

${ }^{29}$ Misak, C., Truth and the End of Inquiry. A Peircean Account of Truth, Clarendon, Oxford, 1991,
} 
siana que busca una certeza apodíctica, una ciencia primera o básica que proporcione un fundamento claro y distinto, la aproximación pragmatista destaca que el desarrollo histórico de la ciencia ha consistido en una peculiar articulación de falibilismo y objetividad. La experiencia ganada históricamente por la humanidad muestra que el camino mediante el que los seres humanos hemos progresado decididamente hacia la verdad -no ciertamente sin titubeos y fracasos- estriba en el refinamiento de los modos de percibir, en la confianza en la capacidad de la razón propia y de las razones ajenas y en la constante revisión, corrección y mejora de sus resultados. Por así decir, la aproximación pragmatista conjura la 'ilusión' fundacionalista que busca un algoritmo capaz de resolver de una vez por todas nuestros problemas y reconoce que no hay una garantía metafísica de que nuestras creencias o nuestros métodos no vayan a necesitar una revisión en el futuro, pero esto no implica -advierte Putnam ${ }^{30}$ - que no sepamos nada acerca de cómo conducir nuestra investigación, ni implica un escepticismo relativista. Se trata por el contrario de una concepción de la racionalidad genuinamente humana, falible (errare hominum est) pero inclinada como por instinto a alcanzar la verdad.

La concepción de la verdad como tarea comunitaria se encuentra en directa confrontación con quienes conciben la actividad racional como la búsqueda cartesiana de fundamentos. La búsqueda individual de fundamentos es una empresa ilusoria, un sueño de la razón, de la que quizá son engendros monstruosos las perversiones totalitarias de nuestro siglo. Por el contrario, tengo para mi que la aproximación pragmatista ofrece una comprensión de la ciencia como actividad vital, cooperativa y falible, de investigación, de búsqueda en comunidad de la verdad que expresa mucho más fielmente las prácticas efectivas de quienes han dedicado su vida a ese quehacer.

\section{Hilary Putnam (1927-)}

La sabiduría convencional de nuestra profesión suele decir que el problema de la verdad fue satisfactoriamente resuelto por Alfred Tarski con su teoría semántica acerca de la verdad y aquel famoso ejemplo de que "La nieve es blanca" es verdadera, si y sólo si la nieve es blanca. Sin embargo aquella teoría, sin duda de valor sobresaliente para la lógica formal, no tenía realmente nada que ver con la verdad. Se trataba de un procedimiento riguroso para la introducción en lógica del predicado "es verdadero" mediante la distinción entre lenguaje objeto y metalenguaje. "La idea de que los problemas filosóficos en torno a la

$1-2$.

${ }^{30}$ Putnam, H., Pragmatism. An Open Question, Blackwell, Oxford, p. 69. 
noción de verdad han sido solucionados de una vez por todas -ha escrito Putnam $^{31}$ - es simplemente un error. Se trata de un error muy importante, porque si se comete, y especialmente si se apela al trabajo de Alfred Tarski, entonces uno está abocado al engaño de que el mayor problema de la filosofía -el problema de cómo el lenguaje y el pensamiento "enganchan" con el mundo- ha sido resuelto por la moderna lógica matemática". Por el contrario, es preciso afirmarempleando la expresión del lógico Saul Kripke- que no hay un sustituto matemático para la filosofía.

Ese engaño es otro subproducto de la cultura cientista de nuestro siglo, heredada del Círculo de Viena. El propio fracaso del programa funcionalista, que pretendía interpretar los procesos cognitivos humanos como procesos de computación desarrollados por ordenadores singularmente sofisticados, ha puesto de manifiesto la necesidad de un marco conceptual más rico para el estudio del lenguaje, los procesos de comunicación y su articulación con el pensamiento y el mundo. Frente al "realismo científico" que ha imperado en la filosofía angloamericana desde los años cincuenta, Hilary Putnam ha sostenido con vigor un realismo de rostro humano, que tiene sus antecedentes en Wittgenstein y en la tradición pragmatista. El énfasis fundamental se pone en la íntima relación de "lo epistemológico" y "lo ontológico" y en la interconexión vital de teoría y práctica en la búsqueda de la verdad: "Quizá lo más importante que trato de defender es la idea de que los aspectos teóricos y prácticos de la filosofía dependen unos de otros. Dewey escribió en Reconstruction in Philosophy que 'la filosofía se recupera a sí misma cuando cesa de ser un recurso para ocuparse de los problemas de los filósofos y se convierte en un método, cultivado por filósofos, para ocuparse de los problemas de los hombres'. Pienso que los problemas de los filósofos y los problemas de varones y mujeres están conectados, y que es parte de la tarea de una filosofía responsable extraer la conexión" ${ }^{32}$.

En contraste con las pretensiones del realismo científico, que "empieza con la promesa de salvar el mundo y termina por deshumanizarlo" ${ }^{33}$, Putnam sostiene que para llevar a cabo un programa de investigación que proporcione una explicación precisa y coherente de la naturaleza del conocimiento y de la objetividad, esa búsqueda ha de tener un rostro humano, ha de estar anclada en nuestras prácticas efectivas de conocimiento. Esto no significa la renuncia a la

\footnotetext{
${ }^{31}$ Putnam, H., "On Truth", Words and Life, Harvard University Press, Cambridge, MA, 1994, 315.

32 J. Harlan: "Entrevista con Hilary Putnam, Acerca de la mente, el significado y la realidad", Atlántida 4 (1993), 80-81.

${ }^{33}$ Conant, J., "Introduction", en Putnam, H., Realism with a Human Face, Harvard University Press, Cambridge, MA, 1990, xvi.
} 
verdad o su subordinación a un perspectivismo culturalista, sino un énfasis en su contribución al florecimiento humano ${ }^{34}$.

Por eso quizá es preciso abordar, aunque sea sólo brevemente, una última cuestión. Se trata de la distinción entre pluralismo y relativismo, que a veces quizá interesadamente es presentada de modo muy borroso. La defensa del pluralismo no implica una renuncia a la verdad o su subordinación a un perspectivismo culturalista. Al contrario, el pluralismo estriba no sólo en afirmar que hay diversas maneras de pensar acerca de las cosas, sino además en sostener que entre ellas hay —en expresión de Stanley Cavell-maneras mejores y peores, y que mediante el contraste con la experiencia y el diálogo racional los seres humanos somos capaces de reconocer la superioridad de un parecer sobre otro. El pluralismo no relativista sostiene que la búsqueda de la verdad es enriquecedora, porque la verdad es perfeccionamiento.

Por el contrario, la posición relativista que afirma que sólo hay diálogo, que sólo hay diversidad de perspectivas radicalmente inconmensurables, no sólo se autorrefuta en la propia formulación, sino que sacrifica la noción de humanidad al negar la capacidad de efectivo perfeccionamiento y de progreso humano. La finalidad del diálogo es la verdad. Lo que distingue el relativismo del pluralismo es que éste último vive precisamente de la ilusión compartida por descubrir la escondida continuidad entre los saberes particulares. La esperanza de lograr en el futuro mejores resultados se convierte así en la mejor garantía para la prosecución de la investigación. "La verdad es una -afirmaba Juan Pablo $\mathrm{II}^{35}$-, pero se presenta a nosotros de forma fragmentaria a través de los múltiples canales que nos conducen a su cercanía diferenciada. (...) en cuanto ciencias, la filosofía y la teología son también ellas intentos limitados para percibir la unidad compleja de la verdad. Es sumamente importante intentar, por una parte, la búsqueda de una síntesis vital cuya nostalgia nos aguijonea, y por otra, evitar cualquier sincretismo irrespetuoso de órdenes de conocimientos y grados de certeza distintos".

\section{Conclusión}

El contexto humano de la búsqueda científica, desinteresada, comunitaria, afectuosa, es marca distintiva de la verdad. La verdad como objetivo de nuestra búsqueda genuina conduce -ha señalado con valentía Susan Haack- a la tesis

\footnotetext{
${ }^{34}$ Putnam, H., Razón, verdad e historia, Tecnos, Madrid, 1988, p. 13.

35 Juan Pablo II, "El hombre frente a la verdad", en L'Osservatore Romano, Edición semanal, 21 de diciembre 1986, p. 22.
} 
sustantiva de que la falta de integridad intelectual, a largo plazo y en general, impide la búsqueda: "Así como el valor es la virtud por excelencia del soldado, podría decirse, simplificando un poco, que la integridad intelectual es la virtud por excelencia del académico" ${ }^{136}$. Por ello fue para mí un regalo -que agradezco vivamente- la invitación a impartir este seminario en la Universidad Católica de Santa Fe. Desearía terminar estas páginas con unas palabras de Peirce que me parece reflejan bien la gozosa realidad que he podido palpar en estos días de trabajo en este Instituto de Filosofía:

"No llamo ciencia a los estudios solitarios de un hombre aislado. Sólo cuando un grupo de hombres [iy mujeres, por supuesto!], más o menos en intercomunicación, se ayudan y se estimulan unos a otros al comprender un conjunto particular de estudios como ningún extraño puede comprenderlo, llamo a su vida ciencia. No es necesario que todos trabajen sobre el mismo problema, o que todos estén completamente familiarizados con todo lo que el otro tiene necesidad de saber; pero sus estudios deben estar tan estrechamente ligados que cualquiera de ellos pudiera hacerse cargo del problema de cualquier otro después de algunos meses de preparación especial, y que cada uno entendiera bastante minuciosamente en qué consiste cada uno de los trabajos de los otros; de tal modo que al juntarse dos de ellos, estarían completamente informados cada uno de las ideas y del lenguaje que el otro hablara y se sentirían como hermanos" ${ }^{137}$.

A estas hermosas y exigentes palabras sólo puedo añadir otras dos: $\mathrm{Mu}$ chas gracias.

\section{Resumen}

La consideración de la verdad como objeto de la búsqueda científica y vital es posiblemente la manera más adecuada para comprender la tradición pragmatista. Frente al diagnóstico postmoderno de Rorty que aboga por la disolución de la filosofía en la literatura, en la conversación general de la humanidad y frente al fundacionalismo cientista de los herederos del Círculo de Viena en este trabajo se defiende un camino intermedio, con pretensiones más modestas, pero que por estar anclado en la experiencia personal de cada uno y en la experiencia colectiva de la especie humana, afronta mejor el reto de dar razón del progreso histórico de la verdad, el reto de comprender el sentido del crecimiento de los saberes y el proceso de generación de nuevos conocimientos. Se trata de la tradición pragma-

\footnotetext{
${ }^{36}$ Haack, S., "El interés por la verdad: qué significa, por qué importa", en Nicolás, J.A., y Frápolli, M. J. (eds.), Teorias de la verdad en el siglo XX, , p. 58.

${ }^{37}$ Peirce, S.C., "The Nature of Science", MS 1334, Adirondack Summer School Lectures, 1905; trad. de S. F. Barrena, Anuario Filosófico 29 (1996), 1437.
} 
tista que hunde sus raíces en el pensamiento de Charles S. Peirce, cobra vigor en nuestro siglo con las reflexiones de Ludwig Wittgenstein y adquiere su mayor desarrollo en autores contemporáneos como Hilary Putnam y Susan Haack.

\begin{abstract}
The consideration of the truth as object of the scientific and vital search is possibly the most adequate way to understand the pragmatist tradition. As opposed to the Rorty's postmodern diagnosis that pleads for the dissolution of the philosophy in the literature or in the general conversation of the humanity; as opposed to the scientist foundation of the inheritors of the Circle of Vienna, in this work an intermediate way with more modest pretensions is presented. Being anchored in the personal experience and in the collective experience of the human, it faced better the challenge of giving reason to the historical progress of the truth and the challenge of understanding the sense of the growth and the generation process of new knowledge. The pragmatist tradition which sinks its roots in Charles's $S$ Peirce's thought., increases vigor in our century with Ludwig Wittgenstein's reflections and acquires its major development in contemporary authors such as Hilary Putnam and Susan Haack.
\end{abstract}

